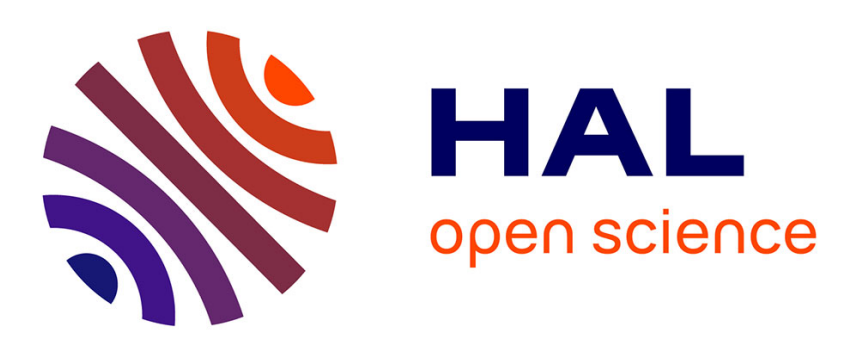

\title{
Transition from Asynchronous to Oscillatory Dynamics in Balanced Spiking Networks with Instantaneous Synapses
}

\author{
Matteo Di Volo, Alessandro Torcini
}

\section{- To cite this version:}

Matteo Di Volo, Alessandro Torcini. Transition from Asynchronous to Oscillatory Dynamics in Balanced Spiking Networks with Instantaneous Synapses. Physical Review Letters, 2018, 121 (12), 10.1103/PhysRevLett.121.128301 . hal-03118109

\author{
HAL Id: hal-03118109 \\ https://hal.science/hal-03118109
}

Submitted on 21 Jan 2021

HAL is a multi-disciplinary open access archive for the deposit and dissemination of scientific research documents, whether they are published or not. The documents may come from teaching and research institutions in France or abroad, or from public or private research centers.
L'archive ouverte pluridisciplinaire HAL, est destinée au dépôt et à la diffusion de documents scientifiques de niveau recherche, publiés ou non, émanant des établissements d'enseignement et de recherche français ou étrangers, des laboratoires publics ou privés. 


\title{
Transition from Asynchronous to Oscillatory Dynamics in Balanced Spiking Networks with Instantaneous Synapses
}

\author{
Matteo di Volo ${ }^{1}$ and Alessandro Torcini ${ }^{2}$ \\ ${ }^{1}$ Unité de Neuroscience, Information et Complexité (UNIC), CNRS FRE 3693, 1 avenue de la Terrasse, 91198 Gif sur Yvette, France \\ ${ }^{2}$ Laboratoire de Physique Théorique et Modélisation, Université de Cergy-Pontoise, \\ CNRS, UMR 8089, 95302 Cergy-Pontoise cedex, France, \\ Max Planck Institut für Physik komplexer Systeme, Nöthnitzer Str. 38, 01187 Dresden, Germany \\ and CNR_Consiglio Nazionale delle Ricerche-Istituto dei Sistemi Complessi, \\ via Madonna del Piano 10, 50019 Sesto Fiorentino, Italy
}

(Received 11 May 2018; revised manuscript received 10 August 2018; published 17 September 2018)

\begin{abstract}
We report a transition from asynchronous to oscillatory behavior in balanced inhibitory networks for class I and II neurons with instantaneous synapses. Collective oscillations emerge for sufficiently connected networks. Their origin is understood in terms of a recently developed mean-field model, whose stable solution is a focus. Microscopic irregular firings, due to balance, trigger sustained oscillations by exciting the relaxation dynamics towards the macroscopic focus. The same mechanism induces in balanced excitatory-inhibitory networks quasiperiodic collective oscillations.
\end{abstract}

DOI: 10.1103/PhysRevLett.121.128301

Introduction.-Cortical neurons fire quite irregularly and with low firing rates, despite being subject to a continuous bombardment from thousands of presynaptic excitatory and inhibitory neurons [1]. This apparent paradox can be solved by introducing the concept of a balanced network, where excitation and inhibition balance each other and the neurons are kept near their firing threshold [2]. In this regime spikes, representing the elementary units of information in the brain, are elicited by stochastic fluctuations in the net input current yielding an irregular microscopic activity, while neurons can promptly respond to input modifications [3].

In neural network models balance can emerge spontaneously in coupled excitatory and inhibitory populations thanks to the dynamical adjustment of their firing rates [4-9]. The usually observed dynamics is an asynchronous state characterized by irregular neural firing joined to stationary firing rates $[4,6,7,9]$. The asynchronous state has been experimentally observed both in vivo and in vitro $[10,11]$; however this is not the only state observable during spontaneous cortical activity. In particular, during spontaneous cortical oscillations excitation and inhibition wax and wane together [12], suggesting that balancing is crucial for the occurrence of these oscillations with inhibition representing the essential component for the emergence of the synchronous activity $[13,14]$.

The emergence of collective oscillations (COs) in inhibitory networks has been widely investigated in networks of spiking leaky integrate-and-fire (LIF) neurons. In particular, it has been demonstrated that COs emerge from asynchronous states via Hopf bifurcations in the presence of an additional timescale, beyond the one associated with the membrane potential evolution, which can be the transmission delay [5,15] or a finite synaptic time [16]. As the frequency of the COs is related to such external timescale this mechanism is normally related to fast $(>30 \mathrm{~Hz})$ oscillations. Nevertheless, despite many theoretical studies, it remains unclear which other mechanisms could be invoked to justify the broad range of COs' frequencies observed experimentally [17].

In this Letter we present a novel mechanism for the emergence of COs in balanced spiking inhibitory networks in the absence of any synaptic or delay timescale. In particular, we show for class I and II neurons [18] that COs arise from an asynchronous state by increasing the network connectivity (in-degree). Furthermore, we show that the COs can survive only in the presence of irregular spiking dynamics due to the dynamical balance. The origin of COs can be explained by considering the phenomenon at a macroscopic level; in particular, we extend an exact meanfield formulation for the spiking dynamics of quadratic integrate-and-fire (QIF) neurons [19] to sparse balanced networks. An analytic stability analysis of the mean-field model reveals that the asymptotic solution for the macroscopic model is a stable focus and determines the frequency of the associated relaxation oscillations. The agreement of this relaxation frequency with the COs' one measured in the spiking network suggests that the irregular microscopic firings of the neurons are responsible for the emergence of sustained COs corresponding to the relaxation dynamics towards the macroscopic focus. This mechanism elicits COs through the excitation of an internal macroscopic timescale, which can range from seconds to tens of milliseconds, yielding a broad range of collective 
oscillatory frequencies. We then analyze balanced excitatory-inhibitory populations revealing the existence of COs characterized by two distinct frequencies, whose emergence is due, also in this case, to the excitation of a meanfield focus induced by fluctuation-driven microscopic dynamics.

The model.-We consider a balanced network of $N$ pulse-coupled inhibitory neurons, whose membrane potential evolves as

$$
\tau_{m} \dot{v}_{i}=F\left(v_{i}\right)+I-2 \tau_{m} g \sum_{j \in \operatorname{pre}(i)} \varepsilon_{i j} \delta\left(t-t_{j}\right)
$$

where $I$ is the external dc current, $g$ is the inhibitory synaptic coupling, $\tau_{m}=20 \mathrm{~ms}$ is the membrane time constant and fast synapses (idealized as $\delta$ pulses) are considered. The neurons are randomly connected, with in degrees $k_{i}$ distributed according to a Lorentzian PDF peaked at $K$ and with a half width at half maximum (HWHM) $\Delta_{K}$. The elements of the corresponding adjacency matrix $\varepsilon_{i j}$ are one (zero) if the neuron $j$ is connected (or not) to neuron $i$. We consider two paradigmatic models of spiking neuron: the quadratic-integrate and fire (QIF) with $F(v)=v^{2}$ [20], which is a current-based model of class I excitability; and the Morris-Lecar (ML) [21,22], representing a conductance-based class II excitable membrane. The dc current and the coupling are rescaled with the median in degree as $I=\sqrt{K} I_{0}$ and $g=g_{0} / \sqrt{K}$, as usually done in order to achieve a self-sustained balanced state for sufficiently large in degrees $[4,6-9,23,24]$. Furthermore, in analogy with Erdös-Renyi networks we assume $\Delta_{K}=$ $\Delta_{0} \sqrt{K}$. We have verified that the reported phenomena are not related to the peculiar choice of the distribution of the in degrees, namely, Lorentzian, needed to obtain an exact mean-field formulation for the network evolution [19], but that they can be observed also for more standard distributions, like Erdös-Renyi and Gaussian ones (for more details see the Supplemental Material [22] and Ref. [25]).

In order to characterize the network dynamics we measure the mean membrane potential $V(t)=$ $\sum_{i=1}^{N} v_{i}(t) / N$, the instantaneous firing rate $R(t)$, corresponding to the number of spikes emitted per unit of time and per neuron, as well as the population averaged coefficient of variation $C V$ [26] measuring the fluctuations in the neuron dynamics. Furthermore, the level of coherence in the neural activity can be quantified in terms of the following indicator [27]

$$
\rho \equiv\left(\frac{\sigma_{V}^{2}}{\sum_{i=1}^{N} \sigma_{i}^{2} / N}\right)^{1 / 2}
$$

where $\sigma_{V}$ is the standard deviation of the mean membrane potential, $\sigma_{i}^{2}=\left\langle V_{i}^{2}\right\rangle-\left\langle V_{i}\right\rangle^{2}$ and $\langle\cdot\rangle$ denotes a time average. A coherent macroscopic activity is associated with a finite value of $\rho$ (perfect synchrony corresponds to $\rho=1$ ), while an asynchronous dynamics to a vanishingly small

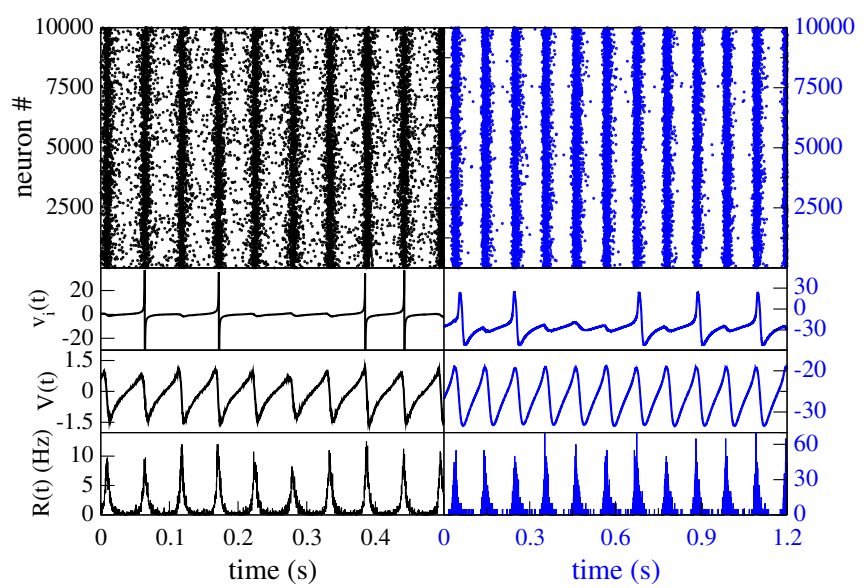

FIG. 1. The panels show (from top to bottom) the raster plots and the corresponding time traces for the membrane potential $v_{i}(t)$ of a representative neuron, for $V(t)$ and $R(t)$. Left row (black): QIF and right row (blue): ML. The parameter values are $N=10000, K=1000, \Delta=0.3, g_{0}=1$, and $I_{0}=0.015$.

$\rho \approx \mathcal{O}(1 / \sqrt{N})$. Time averages and fluctuations are usually estimated on time intervals $\simeq 120 \mathrm{~s}$, after discarding transients $\simeq 2 \mathrm{~s}$.

Results.-In both models we can observe collective firings, or population bursts, occurring at almost constant frequency $\nu_{\text {osc }}$. As shown in Fig. 1, despite the almost regular macroscopic oscillations in the firing rate $R(t)$ and in the mean membrane potential $V(t)$, the microscopic dynamics of the neurons $v_{i}(t)$ is definitely irregular. The latter behavior is expected for balanced networks, where the dynamics of the neurons is driven by the fluctuations in the input current; however usually the collective dynamics is asynchronous and not characterized by $\mathrm{COs}$ as in the present case $[4,6-9,23,24]$.

Asynchronous dynamics is indeed observable also for our models for sufficiently sparse networks (small $K$ ), indeed a clear transition is observable from an asynchronous state to collective oscillations for $K$ larger than a critical value $K_{c}$. As observable from Figs. 2(a), 2(b), where we report the coherence indicator $\rho$ as a function of $K$ for various system sizes from $N=2000$ to $N=20000$. In particular, $\rho$ vanishes as $N^{-1 / 2}$ for $K<K_{c}$ (as we have verified), while it stays finite above the transition, thus indicating the presence of collective motion. This transition resembles those reported for sparse LIF networks with finite synaptic timescales in Refs. [28,29] or with finite time delay in $[5,15]$. However, Poissonian-like dynamics of the single neurons has been reported only in Refs. [5,15].

In the present case, in both the observed dynamical regimes the microscopic dynamics remains quite irregular for all the considered $K$ and system size $N$, as testified by the fact that $C V \simeq 0.8$ for the QIF and $C V \geq 1$ for the ML [as shown in the insets of Figs. 2(a), 2(b)]. The relevance of the microscopic fluctuations for the existence of the collective oscillations in this system can be appreciated 


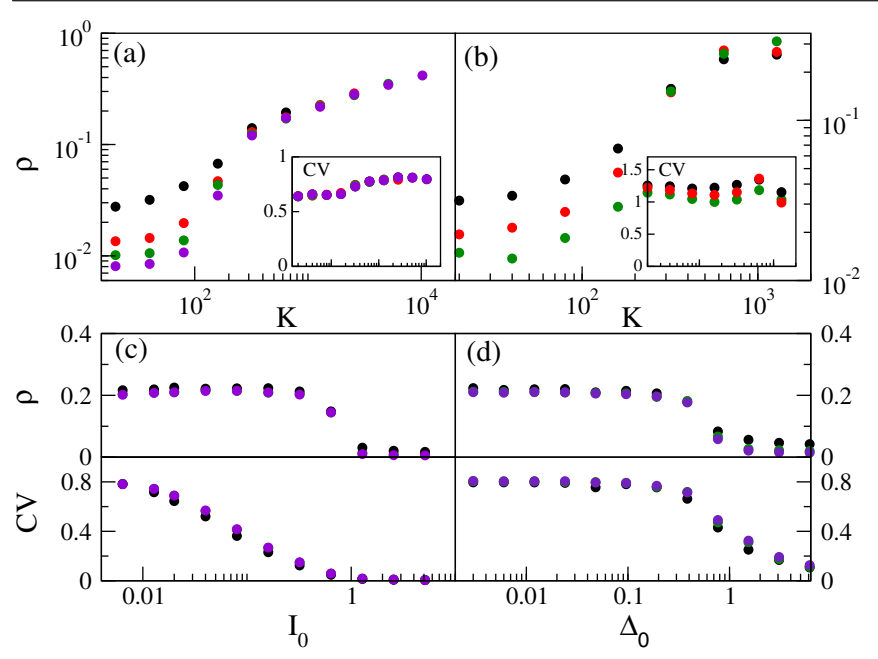

FIG. 2. Upper panels: order parameter $\rho$ versus $K$ for QIF (a) and ML (b), the insets report the corresponding CVs. The lower panels display in the upper part $\rho$ and in the lower one the $C V$ versus $I_{0}$ (c) and $\Delta_{0}$ (d) for the QIF. The data refer to various system sizes: namely, $N=2000$ (black), 5000 (red), 10000 (green), and 20000 (violet). The employed parameters are $I_{0}=0.1, g_{0}=5$, and $\Delta_{0}=1$ for ML (b); for QIF $g_{0}=1$, $\Delta_{0}=0.1, I_{0}=0.006, K=1000$ when not otherwise stated.

by considering the behavior of $\rho$ and $C V$ as a function of the external current $I_{0}$ and of the parameter controlling the structural heterogeneity, namely, $\Delta_{0}$. The results of these analyses are shown in Figs. 2(c) and 2(d) for the QIF and for $N=2000,10000$, and 20000 . In both cases we fixed an in-degree $K>K_{c}$ in order to observe collective oscillations and then we increased $I_{0}$ or $\Delta_{0}$. In both cases we observe that for large $I_{0}\left(\Delta_{0}\right)$ the microscopic dynamics is now imbalanced with few neurons firing regularly with high rates and the majority of neurons suppressed by this high activity. This induces a vanishing of the $C V$, which somehow measures the degree of irregularity in the microscopic dynamics. At large $I_{0}$ the dynamics of the network is controlled by neurons definitely suprathreshold and the dynamics becomes mean driven [30,31]. The same occurs by increasing $\Delta_{0}$, when the heterogeneity in the in-degree distribution becomes sufficiently large only few neurons, the ones with in degrees in proximity of the mean $K$, can balance their activity, while for the remaining neurons it is no more possible to satisfy the balance conditions, as recently shown in Refs. [32-34]. As a result, COs disappear as soon as the microscopic fluctuations, due to the balanced irregular spiking activity, vanish.

Effective mean-field model. - In order to understand the origin of these macroscopic oscillations we consider an exact macroscopic model recently derived in Ref. [19] for fully coupled networks of pulse-coupled QIF with synaptic couplings randomly distributed according to a Lorentzian. The mean-field dynamics of this QIF network can be expressed in terms of only two collective variables (namely, $V$ and $R$ ), as follows [19]:

$$
\dot{R}=\frac{R}{\tau_{m}}\left(2 V+\frac{\Gamma}{\pi}\right), \quad \dot{V}=\frac{V^{2}+I}{\tau_{m}}+R \bar{g}-(\pi R)^{2} \tau_{m},
$$

where $\bar{g}$ is the median and $\Gamma$ the HWHM of the Lorentzian distribution of the synaptic couplings.

Such formulation can be applied to the sparse network studied in this Letter, indeed the quenched disorder in the connectivity can be rephrased in terms of a random synaptic coupling [35]. Namely, each neuron $i$ is subject to an average inhibitory synaptic current of amplitude $g_{0} k_{i} R /(\sqrt{K})$ proportional to its in-degree $k_{i}$. Therefore, we can consider the neurons as fully coupled, but with random values of the coupling distributed as a Lorentzian of median $\bar{g}=-g_{0} \sqrt{K}$ and HWHM $\Gamma=g_{0} \Delta_{0}$. The mean-field formulation (3) takes now the expression,

$$
\begin{gathered}
\tau_{m} \dot{R}=R\left(2 V+\frac{g_{0} \Delta_{0}}{\pi}\right) \\
\tau_{m} \dot{V}=V^{2}+\sqrt{K}\left(I_{0}-\tau_{m} g_{0} R\right)-\left(\pi R \tau_{m}\right)^{2} .
\end{gathered}
$$

As we will verify in the following, this formulation represents a quite good approximation of the collective dynamics of our network. Therefore, we can safely employ such an effective mean-field model to interpret the observed phenomena and to obtain theoretical predictions for the spiking network.

Let us first consider the fixed point solutions $(\bar{V}, \bar{R})$ of Eqs. (4), (5). The result for the average membrane potential is $\bar{V}=\left(-g_{0} \Delta_{0}\right) /(2 \pi)$, while the firing rate is given by the following expression:

$$
\bar{R} \tau_{m}=\frac{g_{0} \sqrt{K}}{2 \pi^{2}}\left(\sqrt{1+\frac{4 \pi^{2}}{\sqrt{K}} \frac{I_{0}}{g_{0}^{2}}+\frac{\Delta_{0}^{2}}{K}}-1\right) .
$$

This theoretical result reproduces quite well with the simulation findings for the QIF spiking network in the asynchronous regime (observable for sufficiently high $\Delta_{0}$ and $I_{0}$ ) over a quite broad range of connectivities (namely, $10 \leq K \leq 10^{4}$ ), as shown in Fig. 3(a). At the leading order in $K$, the firing rate (6) is given by $R_{a} \tau_{m}=I_{0} / g_{0}$, which represents the asymptotic result to which the balanced inhibitory dynamics converges for sufficiently large in degrees irrespectively of the considered neuronal model, as shown in Figs. 3(a) and 3(b) for the QIF and ML models and as previously reported in Ref. [24] for LIF neurons. In particular, for the ML model the asymptotic result $R_{a}$ is attained already for $K \geq 500$, while for the QIF model in degrees larger than $10^{4}$ are required.

The linear stability analysis of the solution $(\bar{V}, \bar{R})$ reveals that this is always a stable focus, characterized by two complex conjugate eigenvalues with a negative real part $\Lambda_{R} \tau_{m}=-\Delta_{0} / 2 \pi$ and an imaginary part 

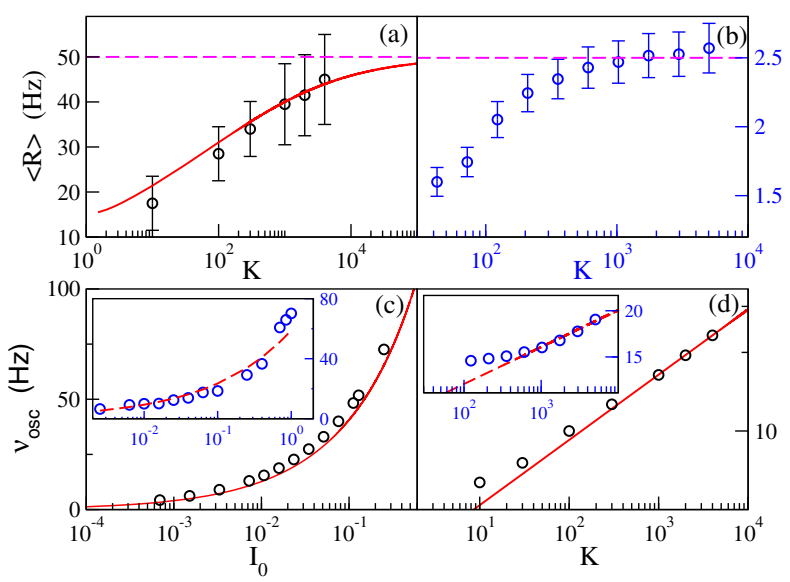

FIG. 3. Upper panels: average firing rates $\langle R\rangle$ versus $K$ for QIF (a) and ML (b), the horizontal dashed (magenta) lines denote $R_{a}$ and the solid (red) line in (a) $\bar{R}$ in Eq. (6). The choice of parameters $\left(I_{0}, \Delta_{0}\right)$ sets the dynamics as asynchronous: $(1,3)$ in (a) and $(0.05,8)$ in (b). Lower panels: $\nu_{\text {osc }}$ versus $I_{0}(\mathrm{c})$ and versus $K$ (d) for the QIF, the insets display the same quantities for the ML. The red solid line in (c) refers to $\nu_{\text {th }}$, and in (d) to the theoretically predicted scaling $\nu_{\text {th }} \sim K^{\frac{1}{4}}$; the red dashed line in the inset of (c) and (d) to power-law fitting $\nu_{\mathrm{osc}} \simeq I^{0.40}$ and $\nu_{\mathrm{osc}} \simeq K^{0.10}$, respectively. Oscillatory dynamics is observable for the selected parameter's values $\left(I_{0}, \Delta_{0}\right):(0.05,0.3)$ in (c) and $(0.05,0.5)$ in (d). Other parameters' values $N=10000, g_{0}=1$, and $K=1000$ in (c).

$\Lambda_{I} \tau_{m}=\sqrt{2 \bar{R} \tau_{m}\left(2 \pi^{2} \bar{R} \tau_{m}+\sqrt{K} g_{0}\right)-\left(\Delta_{0} / 2 \pi\right)^{2}}$. The frequency of the relaxation oscillations towards the stable fixed point solution is given by $\nu_{\text {th }}=\Lambda_{I} / 2 \pi$. This represents a good approximation of the frequency $\nu_{\text {osc }}$ of the sustained collective oscillations observed in the QIF network over a wide range of values ranging from ultraslow rhythms to high $\gamma$ band oscillations, as shown in Fig. 3(c). Furthermore, it can be shown that $\nu_{\text {th }}$ predicts the correcting scaling of $\nu_{\text {osc }}$ for the QIF for sufficiently large dc currents and/or median in-degree $K$, namely, $\nu_{\text {th }} \approx I_{0}^{1 / 2} K^{1 / 4}$ [as shown in Figs. 3(c) and 3(d)]. For the ML we observe similar scaling behaviors for $\nu_{\text {osc }}$, with slightly different exponent, namely, $\nu_{\text {osc }} \approx I_{0}^{0.4}$ and $\nu_{\text {osc }} \simeq K^{0.10}$; however in this case we have no theoretical prediction with which to compare [see the insets of Figs. 3(c) and 3(d)].

Excitatory-inhibitory balanced populations.-So far we have considered only balanced inhibitory networks, but in the cortex the balance occurs among excitatory and inhibitory populations. To verify if also in this case collective oscillations could be identified we have considered a neural network composed of $80 \%$ excitatory QIF neurons and 20\% inhibitory ones (for more details on the considered model see the Supplemental Material in Ref. [22]). The analysis reveals that also in this case collective oscillations can be observed in the balanced network in the presence of irregular microscopic dynamics
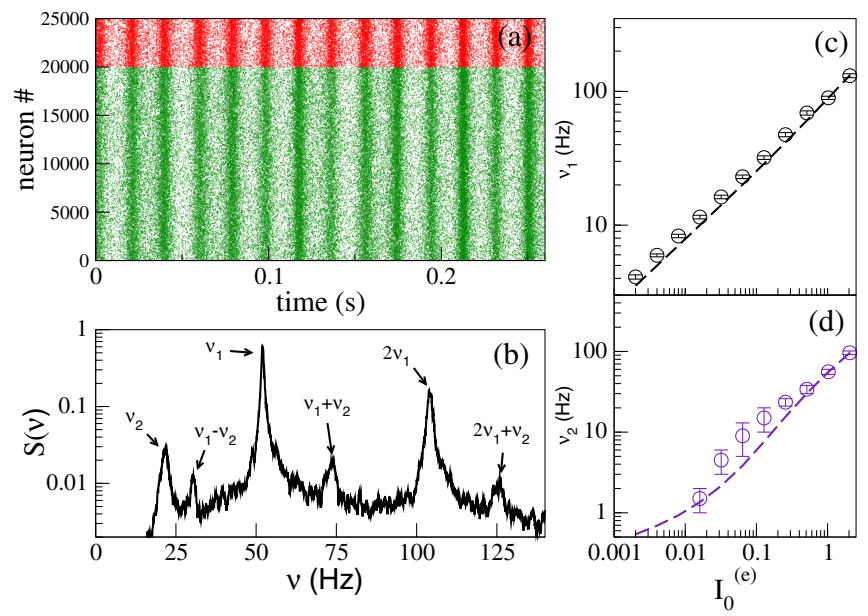

FIG. 4. The raster plot for a network of $N_{E}=20000$ excitatory (green) and $N_{I}=5000$ inhibitory (red) QIF neurons is displayed in (a). In (c) and (d) the COs' frequencies, measured from the power spectrum $S(\nu)$ of the mean voltage $V(t)$ [shown in (b)], are reported as symbols versus the excitatory dc current $I_{0}^{e}$. The dashed lines are the theoretical mean-field predictions. The values of the parameters are reported in Ref. [22].

of the neurons. This is evident from the raster plot reported in Fig. 4(a). An important novelty is that now the oscillations are characterized by two fundamental frequencies as it becomes evident from the analysis of the power spectrum $S(\nu)$ of the mean voltage $V(t)$ shown in Fig. 4(b). As expected for a noisy quasiperiodic dynamics, the spectrum reveals peaks of finite width at frequencies that can be obtained as linear combinations of two fundamental frequencies $\nu_{1}$ and $\nu_{2}$. The origin of the noisy contribution can be ascribed to the microscopic irregular firings of the neurons. Analogously to the inhibitory case, a theoretical prediction for the collective oscillation frequencies can be obtained by considering an effective mean-field model for the excitatory and inhibitory populations of QIF neurons. The model is now characterized by 4 variables, i.e., the mean membrane potential and the firing rate for each population, and also in this case one can find as stationary solutions of the model a stable focus. However, the stability of the focus is now controlled by two couples of complex conjugate eigenvalues; thus the relaxation dynamics of the mean field towards the fixed point is quasiperiodic (see the Supplemental Material for more details [22]). A comparison between the theoretical values of these relaxation frequencies and the measured oscillation frequencies $\nu_{1}$ and $\nu_{2}$ associated to the spiking network dynamics is reported in Figs. 4(c) and 4(d) for a wide range of dc currents, revealing an overall good agreement. Thus suggesting that the mechanism responsible for the collective oscillations remains the same identified for the inhibitory network.

Conclusions.-We have shown that in balanced spiking networks with instantaneous synapses COs can be triggered 
by microscopic irregular fluctuations, whenever the neurons will share a sufficient number of common inputs. Therefore, for a sufficiently large in degree the erratic spiking emissions can promote coherent dynamics. We have verified that the inclusion of a small synaptic timescale does not alter the overall scenario [25].

It is known that heuristic firing-rate models, characterized by a single scalar variable (e.g., the Wilson-Cowan model [36]), are unable to reproduce synchronization phenomena observed in spiking networks [37,38]. In this Letter, we confirm that the inclusion of the membrane dynamics in the mean-field formulation is essential to correctly predict the frequencies of the COs, not only for finite synaptic times (as shown in Ref. [38]), but also for instantaneous synapses in dynamically balanced sparse networks. In this latter case, the internal timescale of the mean-field model controls the COs' frequencies over a wide and continuous range. As we have verified, sustained oscillations can be triggered in the mean-field model by adding noise to the membrane dynamics. Therefore, an improvement of the mean-field theory here presented should include fluctuations around the mean values. A possible strategy could follow the approach reported in Ref. [37] to derive high-dimensional firing-rate models from the associated Fokker-Planck description of the neural dynamics $[5,15]$. Of particular interest would be to understand if a two-dimensional rate equation [37] is sufficient to faithfully reproduce collective phenomena also in balanced networks.

Our results pave the way for a possible extension of the reported mean-field model to spatially extended balanced networks [39-42] by following the approach employed to develop neural fields from neural mass models [43].

The authors acknowledge N. Brunel for extremely useful comments on a preliminary version of this Letter, as well as V. Hakim, E. Montbrió, and L. Shimansky-Geier for enlightening discussions. A. T. has received partial support by the Excellence Initiative I-Site Paris Seine (No. ANR16-IDEX-008) and by the Labex MME-DII (No. ANR-11LBX-0023-01). The work has been mainly realized at the Max Planck Institute for the Physics of Complex Systems (Dresden, Germany) as part of the activity of the Advanced Study Group "From Microscopic to Collective Dynamics in Neural Circuits."

[1] A. Destexhe and D. Paré, J. Neurophysiol. 81, 1531 (1999).

[2] T. P. Vogels, K. Rajan, and L.F. Abbott, Annu. Rev. Neurosci. 28, 357 (2005).

[3] S. Denève and C. K. Machens, Nat. Neurosci. 19, 375 (2016).

[4] C. van Vreeswijk and H. Sompolinsky, Science 274, 1724 (1996).

[5] N. Brunel, J. Comput. Neurosci. 8, 183 (2000).
[6] A. Renart, J. de la Rocha, P. Bartho, L. Hollender, N. Parga, A. Reyes, and K. D. Harris, Science 327, 587 (2010).

[7] A. Litwin-Kumar and B. Doiron, Nat. Neurosci. 15, 1498 (2012).

[8] J. Kadmon and H. Sompolinsky, Phys. Rev. X 5, 041030 (2015).

[9] M. Monteforte and F. Wolf, Phys. Rev. Lett. 105, 268104 (2010).

[10] J. Barral and A. D. Reyes, Nat. Neurosci. 19, 1690 (2016).

[11] N. Dehghani, A. Peyrache, B. Telenczuk, M. Le Van Quyen, E. Halgren, S. S. Cash, N. G. Hatsopoulos, and A. Destexhe, Sci. Rep. 6, 23176 (2016).

[12] M. Okun and I. Lampl, Nat. Neurosci. 11, 535 (2008).

[13] J. S. Isaacson and M. Scanziani, Neuron 72, 231 (2011).

[14] M. Le Van Quyen, L. E. Muller, B. Telenczuk, E. Halgren, S. Cash, N. G. Hatsopoulos, N. Dehghani, and A. Destexhe, Proc. Natl. Acad. Sci. U.S.A. 113, 9363 (2016).

[15] N. Brunel and V. Hakim, Neural Comput. 11, 1621 (1999).

[16] C. Van Vreeswijk, L. Abbott, and G. B. Ermentrout, J. Comput. Neurosci. 1, 313 (1994).

[17] G. Chen, Y. Zhang, X. Li, X. Zhao, Q. Ye, Y. Lin, H. W. Tao, M. J. Rasch, and X. Zhang, Neuron 96, 1403 (2017).

[18] E. M. Izhikevich, Dynamical Systems in Neuroscience (MIT Press, Cambridge, MA, 2007).

[19] E. Montbrió, D. Pazó, and A. Roxin, Phys. Rev. X 5, 021028 (2015).

[20] G. B. Ermentrout and N. Kopell, SIAM J. Appl. Math. 46, 233 (1986).

[21] C. Morris and H. Lecar, Biophys. J. 35, 193 (1981).

[22] See Supplemental Material at http://link.aps.org/ supplemental/10.1103/PhysRevLett.121.128301 for details on the ML model, on inhibitory QIF networks with Gaussian distributed in degrees, on the excitatory-inhibitory QIF network, and the corresponding mean-field model.

[23] S. Jahnke, R.-M. Memmesheimer, and M. Timme, Front. Comput. Neurosci. 3, 13 (2009).

[24] M. Monteforte and F. Wolf, Phys. Rev. X 2, 041007 (2012).

[25] H. Bi, M. Segneri, M. di Volo, and A. Torcini, Coexistence of fast and slow gamma oscillations in a single inhibitory neural population (to be published).

[26] The coefficient of variation $c v(i)$ for the neuron $i$ is the ratio between the standard deviation and the mean of the interspike intervals associated with the train of spikes emitted by the considered neuron. The average coefficient of variation is defined as $C V=\sum_{i} c v(i) / N$.

[27] D. Golomb, Scholarpedia 2, 1347 (2007).

[28] D. Golomb and D. Hansel, Neural Comput. 12, 1095 (2000).

[29] S. Luccioli, S. Olmi, A. Politi, and A. Torcini, Phys. Rev. Lett. 109, 138103 (2012).

[30] A. Renart, R. Moreno-Bote, X.-J. Wang, and N. Parga, Neural Comput. 19, 1 (2007).

[31] D. Angulo-Garcia, S. Luccioli, S. Olmi, and A. Torcini, New J. Phys. 19, 053011 (2017).

[32] I. D. Landau, R. Egger, V. J. Dercksen, M. Oberlaender, and H. Sompolinsky, Neuron 92, 1106 (2016).

[33] R. Pyle and R. Rosenbaum, Phys. Rev. E 93, 040302 (2016).

[34] F. Farkhooi and W. Stannat, Phys. Rev. Lett. 119, 208301 (2017). 
[35] M. di Volo, R. Burioni, M. Casartelli, R. Livi, and A. Vezzani, Phys. Rev. E 90, 022811 (2014).

[36] H. R. Wilson and J. D. Cowan, Biophys. J. 12, 1 (1972).

[37] E. S. Schaffer, S. Ostojic, and L. F. Abbott, PLoS Comput. Biol. 9, e1003301 (2013).

[38] F. Devalle, A. Roxin, and E. Montbrió, PLoS Comput. Biol. 13, e1005881 (2017).

[39] B. Kriener, M. Helias, S. Rotter, M. Diesmann, and G. T. Einevoll, BMC Neurosci. 14, P123 (2013).
[40] R. Rosenbaum and B. Doiron, Phys. Rev. X 4, 021039 (2014).

[41] R. Pyle and R. Rosenbaum, Phys. Rev. Lett. 118, 018103 (2017).

[42] J. Senk, K. Korvasová, J. Schuecker, E. Hagen, T. Tetzlaff, M. Diesmann, and M. Helias, arXiv:1801.06046.

[43] S. Coombes, Scholarpedia 1, 1373 (2006), revision No. 138631. 